\title{
White-matter abnormalities in brain during early abstinence from methamphetamine abuse
}

\author{
Marc C. Tobias • Joseph O'Neill • Matthew Hudkins • \\ George Bartzokis • Andrew C. Dean • Edythe D. London
}

Received: 13 July 2009 / Accepted: 14 December 2009 /Published online: 27 January 2010

(C) The Author(s) 2010. This article is published with open access at Springerlink.com

\begin{abstract}
Background Previous studies revealed microstructural abnormalities in prefrontal white matter and corpus callosum of long-term abstinent chronic methamphetamine abusers. In view of the importance of the early abstinence period in treatment retention, we compared 23 methamphetaminedependent subjects abstinent from methamphetamine for 713 days with 18 healthy comparison subjects. As certain metabolic changes in the brain first manifest after early
\end{abstract}

Preliminary results from this study were presented at the 2008 Society for Neuroscience Annual Meeting, Washington, DC, November 1321, 2008.

M. C. Tobias · J. O’Neill $\cdot$ M. Hudkins $\cdot$ G. Bartzokis $\cdot$

A. C. Dean $\cdot$ E. D. London

Semel Institute for Neuroscience and Biobehavioral Studies,

University of California Los Angeles,

Los Angeles, CA, USA

J. O’Neill $(\square)$

Division of Child \& Adolescent Psychiatry,

Semel Institute for Neurosciences,

University of California Los Angeles,

760 Westwood Plaza \#58-227A,

Los Angeles, CA 90024-1759, USA

e-mail: joneill@mednet.ucla.edu

G. Bartzokis $\cdot$ E. D. London

Brain Research Institute, University of California Los Angeles,

Los Angeles, CA, USA

E. D. London

Department of Molecular and Medical Pharmacology,

University of California Los Angeles,

Los Angeles, CA, USA

G. Bartzokis

Greater Los Angeles VA Healthcare System,

Los Angeles, CA, USA abstinence from methamphetamine, it is also possible that microstructural white-matter abnormalities are not yet present during early abstinence.

Methods Using diffusion tensor imaging at $1.5 \mathrm{~T}$, fractional anisotropy (FA) was measured in prefrontal white matter at four inferior-superior levels parallel to the anterior commissure-posterior commissure (AC-PC) plane. We also sampled FA in the corpus callosum at the midline and at eight bilateral, fiber-tract sites in other regions implicated in effects of methamphetamine.

Results The methamphetamine group exhibited lower FA in right prefrontal white matter above the AC-PC plane $(11.9 \%$ lower; $p=0.007)$, in midline genu corpus callosum $(3.9 \% ; p=0.019)$, in left and right midcaudal superior corona radiata $(11.0 \%$ in both hemispheres, $p$ 's $=0.020$ and 0.016 , respectively), and in right perforant fibers (7.3\%; $p=0.025)$. FA in left midcaudal superior corona radiata was correlated with depressive and generalized psychiatric symptoms within the methamphetamine group.

Conclusions The findings support the idea that methamphetamine abuse produces microstructural abnormalities in white matter underlying and interconnecting prefrontal cortices and hippocampal formation. These effects are already present during the first weeks of abstinence from methamphetamine and are linked to psychiatric symptoms assessed during this period.

Keywords Diffusion tensor imaging (DTI) .

Methamphetamine - Frontal white matter C Corpus callosum . Perforant path · Psychiatric symptoms

As methamphetamine (MA) abuse is among the world's fastest growing illicit drug problems (Rawson and Condon 2007; United Nations Office on Drug and Crime 2003), 
neuroimaging has been applied to delineate possible effects of MA abuse on the brain (reviewed in Barr et al. 2006; Aron et al. 2007; Baicy and London 2007; Chang et al. 2007; Berman et al. 2008; Payer and London 2009). Human and animal studies consistently find deficits related to dopamine function following chronic MA administration, particularly in striatum and prefrontal cortex (Wilson et al. 1996; McCann et al. 1998; Volkow et al. 2001; Sekine et al. 2003). Toxic damage to these and other brain regions may appear as tissue atrophy or hypertrophy, and several groups, including our own (Berman et al. 2008; Thompson et al. 2004), have noted structural abnormalities in cerebral cortex, nuclear gray matter, and white matter of human subjects who had abused MA.

In vivo comparisons of the brains of subjects who had abused MA chronically to those of healthy control (HC) subjects show larger white-matter volume (Thompson et al. 2004; Bartzokis et al. 2000; Chang et al. 2005), abnormal tract morphology (Oh et al. 2005), lower levels of the neuron-associated amino acid $N$-acetyl-aspartate (Ernst et al. 2000), higher levels of the glial-associated sugar myoinositol (mI; Sung et al. 2007), lower glucose metabolism (Kim et al. 2005), and higher incidence of white-matter signal hyperintensities (Bae et al. 2006; Berman et al. 2008, review). These effects were seen in frontal, temporal, and occipital lobes and in the corpus callosum. Three studies used diffusion tensor imaging (DTI), an magnetic resonance imaging (MRI)-based technique that probes whitematter microstructure (Basser 1995) and showed lower fractional anisotropy (FA) in prefrontal white matter (Chung et al. 2007) and genu of corpus callosum (Salo et al. 2008; Kim et al. 2009) in MA-abusing than in HC subjects. FA in the MA subjects was correlated with performance on the Wisconsin Card Sorting and Stroop Interference tasks. These studies targeted volumes-ofinterest (VOIs) within the frontal lobes (Chung et al. 2007) or corpus callosum (Salo et al. 2008; Kim et al. 2009) and examined subjects who had been abstinent from MA for 18 days-10 years, suggesting that the observed abnormalities are long lasting.

Our research team has shown increases in glucose metabolism in parietal cortices between the first and fourth weeks of abstinence from chronic MA abuse (Berman et al. 2007), extending a prior finding of abnormally high parietal cortical glucose metabolism in long-term abstinent MAabusing subjects (Volkow et al. 2001). The latter finding was interpreted as evidence of gliosis, which would be consistent with elevated cerebral glucose metabolism (Roh et al. 1998). Notably, exposure of rodents to MA induces gliosis in brain (Escubedo et al. 1998; Sheng et al. 1994). Increased cortical glucose metabolism during the first month of abstinence from MA, therefore, suggests that gliosis arises and/or increases during this period (Berman et al. 2007). As mI, abundant in glial cells (Brand et al. 1993), is elevated in white matter during long-term abstinence from MA abuse (Sung et al. 2007), gliosis may occur in white matter as well. In study rationale, we asked whether white-matter gliosis, or axonal or myelin damage, was present in the first weeks of abstinence, a critical time for establishing engagement in therapy, and thus treatment outcome (Brecht et al. 2000). Identification of white-matter pathology in this time period might be useful in patient management or prognosis.

As one possible index of gliosis, we chose to measure DTIderived FA. Whereas healthy white matter has high directional diffusion and therefore high FA, gliosis is nondirectionally oriented and hence should reduce FA of local tissue (Jones et al. 1999). Abnormally low FA has been observed in human ischemic white-matter signal hyperintensities, lesions which show gliosis (Jones et al. 1999). Similar inferences have associated low FA with gliosis in dorsal posterior cingulate in patients with Alzheimer's disease (Ding et al. 2008) and in spinal cord (Agosta et al. 2007) and periventricular whitematter signal hyperintensities (Bastin et al. 2009) related to normal aging. Within-subject correlations between low FA and reactive gliosis have been verified in mice (Ahrens et al. 1998) and in resected white matter from human epileptic patients (Dumas de la Roque et al. 2005). Nonetheless, FA and gliosis were not correlated in postmortem multiple sclerosis brains (Schmierer et al. 2008), and pathology other than gliosis (e.g., myelin and/or axonal damage) may also reduce white-matter FA. In particular in primates, exposure to amphetamines may lead to both proximal and terminal serotonin axonal damage following methamphetamine exposure (reviewed in Ricaurte and McCann 1992; Huether et al. 1997).

We measured DTI-derived FA in several white-matter regions in MA-dependent and $\mathrm{HC}$ subjects, making measurements in large $\left(9 \times 9 \times 3 \mathrm{~mm}^{3}\right)$ axial-oblique whitematter slabs at four inferior-superior levels deep to bilateral prefrontal cortex, as in (Chung et al. 2007). In order to assign putative effects of MA to individual white-matter tracts connecting with frontal cortex, we also sampled FA in single DTI voxels at multiple sites along the corpus callosum, corona radiata, and internal capsule. Moreover, as we previously found gray-matter volumetric deficits in hippocampus (Thompson et al. 2004), we sampled single DTI voxels along the perforant path of the hippocampal formation, to determine whether white matter innervating damaged hippocampal cortex is abnormal.

About $50 \%$ of adults with amphetamine dependence experience clinical major depression (Conway et al. 2006). Major depression and depressive symptoms are prominent in the first 3 weeks of abstinence from MA abuse (McGregor et al. 2005) and have been related to glucose metabolism in pregenual anterior cingulate and amygdala 
in early abstinence from MA abuse (London et al. 2004). Other psychiatric symptoms that are common in MAabusing subjects include psychosis, anxiety, suicidal ideation, and hostility (Leventhal et al. 2008; Kalechstein et al. 2000; Drake et al. 2008). Generalized positive psychiatric symptoms have been associated with metabolites measured with proton magnetic resonance spectroscopy (Sekine et al. 2002) and with dopamine transporter availability (Sekine et al. 2001) in striatum. We therefore also tested whether regional white-matter microstructural integrity (FA) was related to depressive and generalized psychiatric symptoms.

\section{Methods and materials}

\section{Subjects}

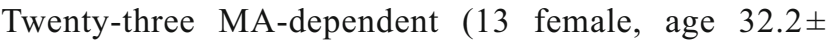
9.7 years) and $18 \mathrm{HC}$ (seven female, $33.3 \pm 8.4$ years) subjects were recruited for the study, which was approved by the University of California Los Angeles (UCLA) institutional review board. Subjects were recruited through local print and radio advertisements and gave written informed consent after receiving a detailed explanation of the study. They were paid volunteers. Inclusion requirements were as follows: at least moderate intelligence (score $>85$ on the Shipley Institute of Living Scale; Zachary et al. 1985), right-handedness (score $>20$ on a modified Edinburgh inventory; Oldfield 1971), absence of current axis I psychiatric disorders (other than nicotine dependence for all subjects and MA dependence for MA subjects), and regular use of tobacco (cigarettes). The MA and HC groups did not differ significantly in years of smoking or nicotine dependence. Exclusionary criteria were significant head trauma with loss of consciousness for any length of time; neurological, cardiovascular, or pulmonary disease; HIV seropositivity, and other significant medical conditions. Subjects who reported mild marijuana $(<1$ cigarette per week) and/or alcohol ( $<14$ drinks per week) consumption were permitted in both groups.

Participants in both groups were administered the Structured Clinical Inventory for DSM-IV Axis I Disorders (First et al. 1995), which was used to establish axis I psychiatric diagnoses. A current axis I diagnosis other than nicotine dependence and MA dependence for the MAdependent group was exclusionary. MA subjects satisfied DSM-IV criteria for amphetamine dependence (with MA specified as the amphetamine that was used; First et al. 1995), and MA use within 3 to 4 days of enrollment was verified with a positive urine screen at enrollment. The MA subjects stayed at the UCLA General Clinical Research Center inpatient unit for the duration of the study. Urine tests were conducted randomly to ensure abstinence. DTI for MA subjects was acquired within 4-10 days of admission (i.e., 7-13 days after last MA use). HC subjects participated on a nonresidential basis and had to demonstrate a urine screen negative for MA, other drugs of abuse, and relevant metabolites on day of scan.

Subjects were administered the Beck Depression Inventory (BDI; Beck et al. 1961) and the Brief Symptom Inventory (BSI; Derogatis and Melisaratos 1983) around the time of MR scanning in the case of MA subjects within 7 days of admission. Two MA subjects had BDI administered and one of these also had BSI administered outside this timeframe. As correlation results involving BDI and BSI remained statistically significant when these two subjects were excluded from analysis, their data were included. BDI score was used as a measure of depressive symptoms. The BSI Positive Symptom Total score was used as a global measure of psychiatric symptoms.

\section{MR image acquisition and processing}

DTI acquisition and postprocessing have been described (Ringman et al. 2007). Data were acquired on a Siemens Sonata 1.5-T MR scanner. High-resolution, T1-weighted sagittal whole-brain structural MRI was used for positioning. Four sequential, six-direction $[(1,0,1),(-1,0,1)$, $(0,1,1),(0,1,-1),(1,1,0),(-1,1,0)]$ diffusion-weighted whole-brain volumes were acquired using echo-planar imaging (repetition time/echo time $=6,000 / 78 \mathrm{~ms}$ ) parallel to the anterior commissure-posterior commissure (AC-PC) plane. The $b$ values were 0 and $1,000 \mathrm{~s} / \mathrm{mm} 2$ with nominal voxel size $3 \times 3 \times 3 \mathrm{~mm}^{3}$. For each subject, the four volumes were coregistered off-line using FMRIB's Linear Image Registration Tool (Jenkinson and Smith 2001) and averaged together. Eddy current correction was applied using FMRIB's Diffusion Toolbox in FSL version 3.2 (Behrens et al. 2003; Smith et al. 2004). FA maps were created from the eddy-current corrected file with FSL DTIFIT.

DTI fractional anisotropy: calculation within VOIs, sampling at fiber-tract sites

Two methods were used to extract regional FA from the scan data. The first method was similar to one described before (Chung et al. 2007). FA values were taken from and averaged across each of four axial-oblique (AC-PCaligned) slab VOIs in each frontal lobe. Each VOI was $3 \times 3$ DTI voxels square in-plane and 1 voxel deep. Each VOI was centered within prefrontal white matter and positioned to avoid contamination from neighboring gray matter. Within these constraints, the VOI position yielding the highest average FA value was selected. The four DTI slabs on which the VOIs were selected were as follows: (1) 
the lowest slab was centered $6 \mathrm{~mm}$ below the $\mathrm{AC}-\mathrm{PC}$ plane, (2) the next slab was at the AC-PC plane, (3) the third was $6 \mathrm{~mm}$ above the AC-PC plane, and (4) the final slab was $9 \mathrm{~mm}$ above (Fig. 1). Special care was taken to exclude the corpus callosum, identified with the help of FA color maps indicating the direction of fiber tracts.

The second method, previously described by Ringman et al. (2007), was used to sample DTI voxels at the following 16 fiber-tract VOIs: midline genu and midline splenium of corpus callosum; bilateral anterior, midcaudal, and caudal superior corona radiata; anterior and posterior limbs and genu of the internal capsule; and perforant path of the hippocampal formation. For each structure, the two highest adjacent FA values were recorded and averaged, whereby FA color maps indicating fiber-tract direction were used to supplement voxel selection. Anatomic voxel selection for genu and splenium corpus callosum, anterior corona radiata, and perforant path was as described (Ringman et al. 2007). The superior corona radiata appeared on superior brain slices as a thick inferior-superior tract just lateral to the lateral ventricle in each hemisphere. Voxels were selected from midcaudal and caudal cross sections of this tract at the level of the body of the corpus callosum, i.e., the level of the superior portions of the lateral ventricles (Fig. 2). For internal capsule voxels, the axial-oblique slice where the mesial-lateral distance between the genua of left and right internal capsule was minimized was identified by scrolling ventrally through the brain volume. Starting from that slice, voxels were selected bilaterally from the anterior limb, genu, and posterior limb of the internal capsule (Fig. 3).

Both protocols were carried out by two investigators (MT, MH) who were blind to subject identity or group assignment. Intrarater reliability scores (intraclass correla- tion coefficients) were $0.97(\mathrm{MT})$ and $0.95(\mathrm{MH})$; interrater reliability was 0.88 .

\section{Statistical analysis}

Based on Chung et al. (2007), we predicted lower FA in MA than in $\mathrm{HC}$ subjects in the four bilateral prefrontal white-matter slab VOIs. At each slab VOI, FA for the two groups was compared using one-way analysis of variance (ANOVA) with Bonferroni correction for multiple comparisons using numbered a priori hypotheses. For bilateral fiber-tract VOIs, repeated-measures analysis of variance (R-ANOVA) was performed on the measure FA with hemisphere and fiber-tract VOI as within-subject factors and diagnosis as between-subject factor. Given a significant main effect of diagnosis on FA in R-ANOVA, post hoc analyses were performed on data from each fiber-tract VOI using one-way ANOVA. For the two midline fiber-tract VOIs (genu and splenium corpus callosum), univariate ANOVA was used with diagnosis as between-subject factor.

Although the two groups did not differ significantly in sex, there was a preponderance of females in the MA group, and effects of sex on frontal white-matter FA have been reported (Chung et al. 2007). Therefore, for those VOIs showing a significant effect of diagnosis on FA, ANOVA was repeated comparing corresponding male-tomale and female-to-female subgroups.

Effects of diagnosis on BDI and BSI Positive Symptom Total scores were determined by independent $T$ test. At VOIs showing a significant effect of diagnosis on FA, Spearman correlations were performed between FA and BDI and between FA and BSI Positive Symptom Total within the MA and HC groups. The criterion for statistical significance for all tests was $p<0.05$ two-tailed.
Fig. 1 DTI FA whole-brain color map of an experimental subject. In the left, an axial-oblique (at the AC-PC plane) section. The white box in left prefrontal white matter (PFWM) indicates the boundaries of a 3-mm deep $9 \times 9-\mathrm{mm}^{2}$ slab across which FA values were sampled and averaged in postprocessing. A similar average was obtained for the right PFWM. In the right, a parasagittal section of the same color map depicts the position of the four parallel prefrontal white-matter slabs (white bars) located at the level of the ACPC plane $(C), 6 \mathrm{~mm}$ below $(D)$, and $6(B)$ and $9(A) \mathrm{mm}$ above. Left and right PFWM FA averages were obtained at each level
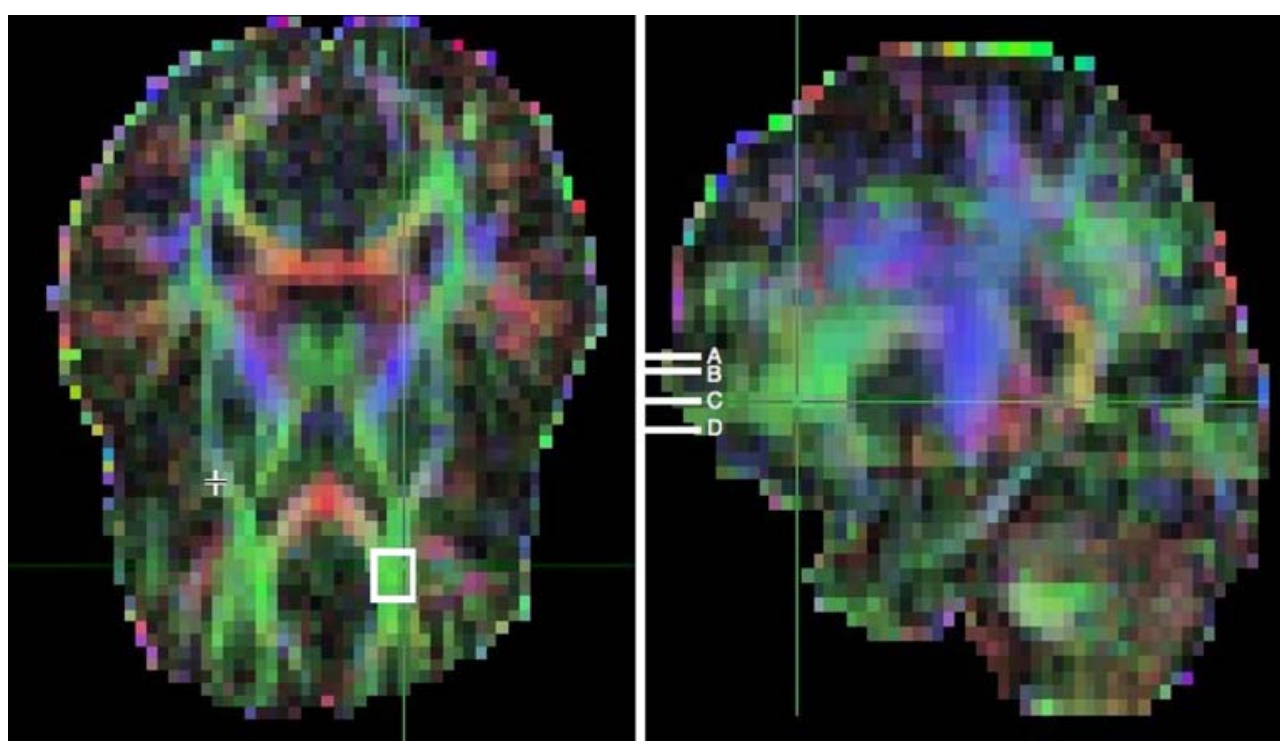
Fig. 2 Axial-oblique (parallel to AC-PC plane) DTI FA brain map at level of the upper lateral ventricles from a study subject. The same image is shown in both left and right. In the left, the green arrow indicates typical site of DTI voxel selection for the midcaudal cross section of the right superior corona radiata white-matter region. In the right, the green arrow indicates typical site for the caudal cross section of the right superior corona radiata

Fig. 3 Axial-oblique (parallel to AC-PC plane) DTI FA brain map at level of the basal ganglia from a selected subject. The same image is shown in all three panels. In the upper left, the green arrow indicates typical site of DTI voxel selection for the anterior limb of the right internal capsule white-matter tract. At the upper right, the arrow indicates the genu of the right internal capsule. At bottom left, the arrow indicates the posterior limb of the right internal capsule
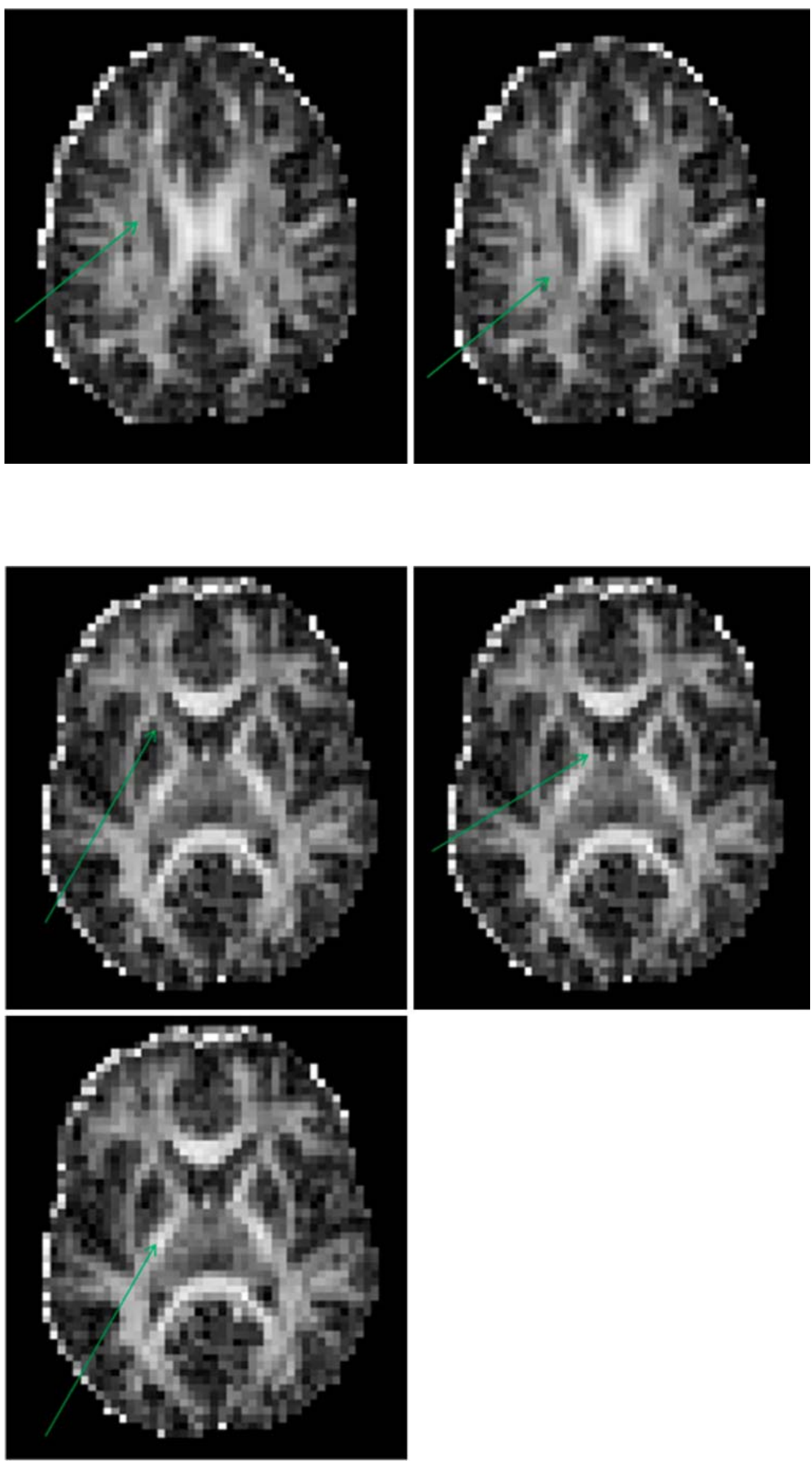


\section{Results}

Subjects

The two subject groups (Table 1) did not differ significantly in sex $\left(\chi^{2}=2.05, p=0.16\right)$, age $(t(38.6)=0.37, p=0.72)$, or alcohol consumption $(t(28.4)=1.08, p=0.29)$. All subjects were smokers and the groups did not differ significantly in years of smoking $(t(33.7)=0.51, p=0.61)$ or scores on the Fagerström Test for Nicotine Dependence $(t(40)=1.32$, $p=0.20)$.

Fractional anisotropy in regional brain white matter

Mean regional values of FA for the MA and $\mathrm{HC}$ groups are shown in Tables 2 and 3. Table 2 includes the prefrontal slab VOIs. A priori hypotheses were formulated for these VOIs based on a prior report (Chung et al. 2007). Table 3 includes the single-voxel fiber-tract sites, for which no a priori hypotheses were formulated but rather omnibus R-ANOVA was performed. FA was lower in MA than in $\mathrm{HC}$ subjects in all eight prefrontal white-matter slab VOIs studied, but the difference was only significant in right prefrontal white matter $6 \mathrm{~mm}$ superior to AC-PC (11.9\%; $F(1,35)=8.0, p=0.007$; Fig. 4). R-ANOVA across the 16 single-voxel white-matter tract sites yielded a significant effect of diagnosis on FA $(F(1,37)=5.4, p=0.026$; Table 3 ) with lower FA in MA than in HC subjects at 12 of 16 sites. In protected post hoc one-way ANOVAs following RANOVA comparisons, the difference was significant only in genu corpus callosum $(3.9 \% ; F(1,40)=6.0, p=0.019)$, left $(11.0 \% ; F(1,40)=5.9, p=0.020)$ and right $(11.0 \% ; F(1$, $40)=6.4, p=0.016$, Fig. 4) midcaudal superior corona radiata, and right perforant path $(7.3 \% ; F(1,40)=5.4$, $p=0.025)$. FA at the midline genu corpus callosum site decreased significantly with years of MA abuse (Spearman, $r=-0.44, p=0.036$ ), but the correlation was not significant at other sites.

Effects of sex on white-matter fractional anisotropy

Chung et al. (2007) observed effects of sex on white-matter FA within their MA sample. Therefore at each site where the between-group difference in FA was significant, we reexamined the difference for the separate male and female subgroups. Among female subjects, FA was significantly lower for MA than for $\mathrm{HC}$ subjects in right prefrontal white matter $6 \mathrm{~mm}$ superior to the AC-PC $(F(1,19)=5.2, p=$ $0.034)$, genu corpus callosum $(F(1,19)=6.0, p=0.025)$, and left $(F(1,19)=7.2, p=0.015)$ and right $(F(1,19)=6.1, p=$ $0.036)$ midcaudal superior corona radiata, i.e., in all regions except right perforant path. In right perforant path, FA tended to be lower for male MA than for male $\mathrm{HC}$ subjects $(F(1,20)=3.0, p=0.099)$, but there was no significant between-group difference for males in right prefrontal white matter $6 \mathrm{~mm}$ superior to the AC-PC, genu corpus callosum, or midcaudal superior corona radiata.

Correlations between white-matter FA and psychiatric symptoms

Mean BDI score was significantly higher in the MA $(15.4 \pm$ 14.1) than in the $\mathrm{HC}(1.8 \pm 2.5)$ group $(t(38)=3.9, p=$ $0.00013)$; as was the mean BSI Positive Symptom Total score (MA 21.9 \pm 14.5, HC 3.1 $\pm 4.7 ; t(38)=5.0, p=$ $0.0000029)$. In left midcaudal superior corona radiata
Table 1 Characteristics of methamphetamine-dependent and healthy control subjects
$M A$ methamphetamine abuser, $H C$ healthy control, $S D$ standard deviation

\begin{tabular}{|c|c|c|c|c|c|c|c|c|}
\hline \multirow[t]{2}{*}{ Subjects } & \multicolumn{4}{|c|}{ MA group $(n=23)$} & \multicolumn{4}{|c|}{ HC group $(n=18)$} \\
\hline & Mean & $n$ & $\mathrm{SD}$ & $\%$ & Mean & $n$ & $\mathrm{SD}$ & $\%$ \\
\hline Age (years) & 32.2 & & 9.7 & & 33.3 & & 8.4 & \\
\hline Tobacco (cigarettes/day) & 10.5 & & 6.4 & & 16.7 & & 7.0 & \\
\hline Tobacco (years) & 13.4 & & 9.4 & & 13.6 & & 6.9 & \\
\hline Tobacco (Fagerström score) & 3.4 & & 1.9 & & 4.2 & & 1.8 & \\
\hline Alcohol (drinks/week) & 1.8 & & 1.9 & & 2.6 & & 2.4 & \\
\hline MA abuse (g/week) & 3.8 & & 4.0 & & - & & - & \\
\hline MA abuse (years) & 8.0 & & 7.0 & & - & & - & \\
\hline Sex (men) & & 10 & & 43.5 & & 11 & & 61.1 \\
\hline Handedness (right) & & 23 & & 100 & & 18 & & 100 \\
\hline Tobacco users & & 23 & & 100 & & 18 & & 100 \\
\hline Alcohol users & & 11 & & 47.8 & & 12 & & 66.7 \\
\hline Lifetime cannabis & & 9 & & 39.1 & & 1 & & 5.6 \\
\hline Lifetime cocaine & & 5 & & 21.7 & & 0 & & 0 \\
\hline
\end{tabular}


Table 2 Prefrontal FA in methamphetamine-dependent and healthy control subjects

\begin{tabular}{lll}
\hline Volume of interest & MA group $(n=23)$ & HC group $(n=18)$ \\
\hline 6 mm inferior to AC-PC plane, left & $0.431 \pm 0.083$ & $0.434 \pm 0.082$ \\
6 mm inferior to AC-PC plane, right & $0.462 \pm 0.065$ & $0.471 \pm 0.107$ \\
at AC-PC plane, left & $0.478 \pm 0.071$ & $0.498 \pm 0.077$ \\
at AC-PC plane, right & $0.490 \pm 0.045$ & $0.501 \pm 0.090$ \\
6 mm superior to AC-PC plane, left & $0.413 \pm 0.066$ & $0.434 \pm 0.073$ \\
6 mm superior to AC-PC plane, right & $0.409 \pm 0.055^{*}$ & $0.465 \pm 0.071$ \\
9 mm superior to AC-PC plane, left & $0.387 \pm 0.061$ & $0.403 \pm 0.053$ \\
9 mm superior to AC-PC plane, right & $0.398 \pm 0.062$ & $0.426 \pm 0.066$ \\
\hline
\end{tabular}

Data are group means \pm standard deviation

$F A$ fractional anisotropy, $A C-P C$ anterior commissure-posterior commissure, $M A$ methamphetamine abuser, $H C$ healthy control

${ }^{*} p<0.01$ (ANOVA Bonferroni corrected for multiple comparisons with a priori hypotheses)

within the MA group (Fig. 5), FA correlated positively with BDI $(r=0.44, p=0.038)$ and BSI Positive Symptom Total $(r=0.51, p=0.014)$. No significant correlations resulted for the MA group at the other anatomic sites where effects of diagnosis on FA had been observed. No significant correlations resulted for the $\mathrm{HC}$ group at any sites where effects of diagnosis on FA were observed. Because the BSI Positive Symptom Total is comprised of items from several subscales, correlations were calculated between left midcaudal superior corona radiata FA and the scores from the BSI subscales. Significant positive correlations $(p<0.05)$ were found for the depression, somatization, obsessivecompulsive, interpersonal sensitivity, paranoid ideation, and phobic anxiety subscales, but not for psychoticism, anxiety, or hostility $(0.053 \leq p \leq 0.16)$.

\section{Discussion}

Summary of findings

A DTI investigation of brain white matter in adult MA subjects revealed modest (3-12\%) decrements in FA in multiple regions, including right prefrontal white matter above the AC-PC plane, bilateral midcaudal superior corona radiata, genu corpus callosum, and right perforant path. Among MA-dependent subjects, the left midcaudal superior corona radiata showed a positive correlation of FA with standard clinical indices of severity of depressive and general psychiatric positive symptoms. Low FA indicates low tissue integrity and/or organization, possibly due to increased extracellular
Table 3 FA at fiber tract in methamphetamine-dependent and healthy control subjects

Data are group means \pm standard deviation

$F A$ fractional anisotropy, $M A$ methamphetamine abuser, $H C$ healthy control

$* p<0.05$ (protected post hoc one-way ANOVA following omnibus repeated-measures ANOVA)

\begin{tabular}{llcr}
\hline Volume of interest & MA group $(n=23)$ & HC group $(n=18)$ & Effect size \\
\hline Genu corpus callosum & $0.848 \pm 0.048^{*}$ & $0.882 \pm 0.041$ & -0.74 \\
Splenium corpus callosum & $0.879 \pm 0.069$ & $0.900 \pm 0.052$ & -0.33 \\
Left anterior corona radiata & $0.572 \pm 0.060$ & $0.597 \pm 0.074$ & -0.37 \\
Right anterior corona radiata & $0.570 \pm 0.067$ & $0.580 \pm 0.085$ & -0.13 \\
Left midcaudal superior corona radiata & $0.387 \pm 0.050^{*}$ & $0.435 \pm 0.075$ & -0.75 \\
Right midcaudal superior corona radiata & $0.382 \pm 0.060^{*}$ & $0.429 \pm 0.058$ & -0.77 \\
Left caudal superior corona radiata & $0.478 \pm 0.067$ & $0.506 \pm 0.076$ & -0.38 \\
Right caudal superior corona radiata & $0.482 \pm 0.061$ & $0.473 \pm 0.061$ & 0.15 \\
Left internal capsule anterior limb & $0.523 \pm 0.074$ & $0.533 \pm 0.071$ & -0.13 \\
Right internal capsule anterior limb & $0.537 \pm 0.065$ & $0.522 \pm 0.073$ & +0.21 \\
Left genu internal capsule & $0.617 \pm 0.052$ & $0.633 \pm 0.063$ & -0.27 \\
Right genu internal capsule & $0.636 \pm 0.069$ & $0.635 \pm 0.065$ & +0.01 \\
Left internal capsule posterior limb & $0.708 \pm 0.040$ & $0.707 \pm 0.040$ & +0.02 \\
Right internal capsule posterior limb & $0.721 \pm 0.050$ & $0.744 \pm 0.036$ & -0.50 \\
Left perforant path & $0.523 \pm 0.053$ & $0.532 \pm 0.069$ & -0.14 \\
Right perforant path & $0.504 \pm 0.04^{*}$ & $0.543 \pm 0.068$ & -0.70 \\
\hline
\end{tabular}




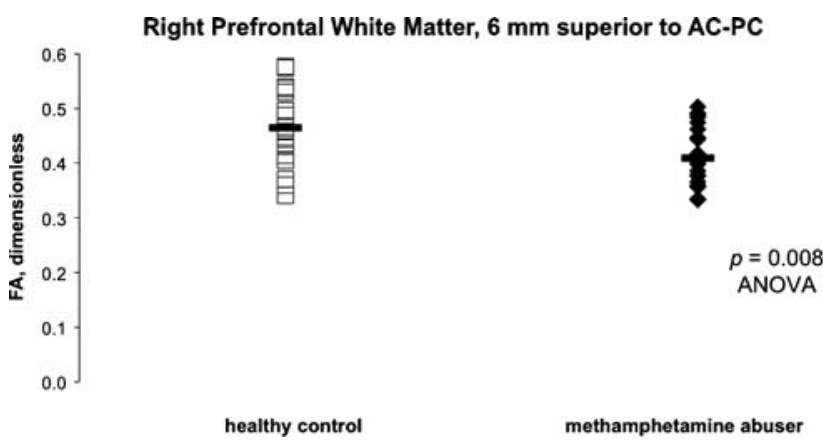

Fig. 4 Key findings for FA obtained in right prefrontal white matter using the methods of Chung et al. (2007; left) and Ringman et al. (2007; right) in MA (solid diamonds) and age-matched HC (open squares) subjects. Group means given by horizontal bars. Mean FA of MA subjects was significantly below that of the HC group in an axial-

water space between fibers and/or deviant course of a portion of the fibers in a voxel (although the latter possibility is reduced in callosal VOIs as those regions contain highly parallel fibers). The results suggest that such aberrant microstructure arises as a neuropathological effect at times of active MA abuse, or may occur in early abstinence from MA abuse. Notably, in rats, three daily exposures to MA are sufficient to induce reactive gliosis lasting 14-21 days (Pennypacker et al. 2000). It is also possible that the white-matter differences seen in our study reflect effects that predated MA abuse. In any case, it is likely that the abnormally low regional FA observed in later remission from MA abuse (Chung et al. 2007; Salo et al. 2008; Kim et al. 2009) has origins in earlier stages of the disease. Specific white-matter pathologies contributing to low FA could include myelin and/or axonal damage and gliosis.

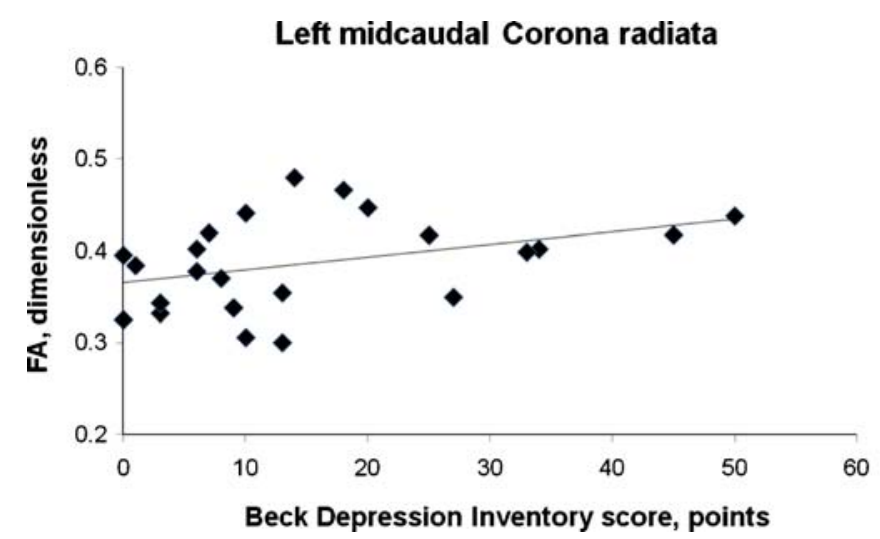

Fig. 5 Relationship of fractional anisotropy $(F A)$ in left midcaudal corona radiata to psychiatric symptoms in MA subjects (solid diamonds). Symptoms were evaluated within 7 days of admission for supervised MA abstinence. Rather than the anticipated negative
Right midcaudal superior Corona radiata

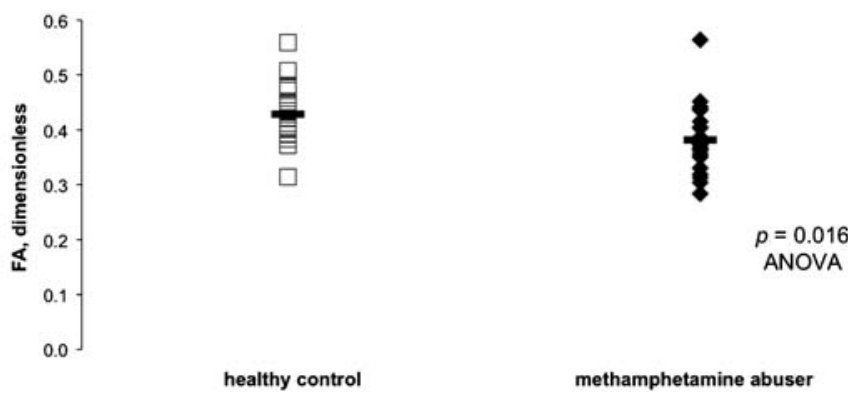

oblique slab of right prefrontal white matter centered $6 \mathrm{~mm}$ above the anterior commissure-posterior commissure $(A C-P C)$ plane. Lower FA of MA subjects than $\mathrm{HC}$ subjects at a midcaudal site along the superior portion of the right corona radiata indicates that this specific tract may be vulnerable to MA abuse

Prefrontal white-matter abnormalities

Our observation of low FA in right prefrontal whitematter superior to the AC-PC plane in the MA group replicates a prior finding (Chung et al. 2007) at $5 \mathrm{~mm}$ above the AC-PC plane. In the earlier report (Chung et al. 2007), the HC subjects in aggregate smoked less tobacco than the MA subjects. In our study, the HC subjects did not smoke less than MA subjects, but the right prefrontal FA finding was similar in both studies. This finding, therefore, is likely associated with MA rather than tobacco abuse. Chung et al. (2007) also observed 7-12\% lower FA in their MA than in their control sample in bilateral prefrontal white matter at the AC-PC level. We observed $2-4 \%$ decrements in FA in our MA group at those sites, but the differences did not reach statistical significance. It is possible that with the higher field strength $(3 \mathrm{~T})$, spatial

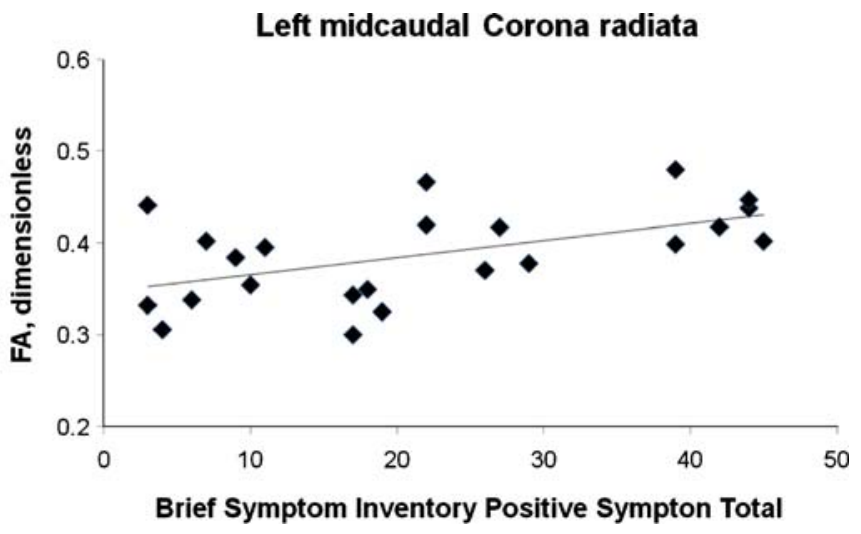

association, FA was significantly positively related to depressive symptoms (BDI score; $r=0.44, p=0.038$ ) and with generalized psychiatric symptoms (Brief Symptom Inventory Positive Symptom Total; $r=0.51, p=0.014$ ) 
resolution $\left(0.9375 \times 0.9375 \times 3.5 \mathrm{~mm}^{3}\right)$, and number of DTI gradients (Berman et al. 2007) used in the prior report (Chung et al. 2007), significant differences might have been evident in our sample as well. These findings reinforce a notion from previous structural MRI (Bae et al. 2006) and magnetic resonance spectroscopy (Ernst et al. 2000; Sung et al. 2007) investigations and studies of cerebral glucose metabolism (Kim et al. 2005) that frontal white matter and possibly other later-myelinating regions are particularly vulnerable to MA abuse. Our measurements of lower FA in the MA than in the healthy control group in corona radiata, however, suggest that additional fiber tracts are involved.

\section{Callosal abnormalities}

At $1.5 \mathrm{~T}$ using six-direction DTI with $1.875 \times 1.875 \times 5 \mathrm{~mm}^{3}$ voxels, Salo et al. (2008) observed $4.8 \%$ lower mean FA (trend) in a VOI encompassing the genu of the corpus callosum in MA-abusing vs. HC subjects. In a VOI encompassing the splenium, they found no significant between-group difference. We detected statistically significant $3.9 \%$ lower FA in the genu and no significant effect in the splenium in the MA group, replicating the findings of Salo et al. (2008) and those of Kim et al. (2009), who recorded highly significant $4.3 \%$ lower FA of the genu in MA subjects. As white-matter fibers passing through the genu interconnect left- and right-hemisphere dorsolateral prefrontal cortices (Pandya and Seltzer 1986), these findings indicate that white-matter innervating prefrontal cortex is particularly involved in the effects of MA abuse. Few (four of 17) HC subjects were tobacco smokers in the Salo study (Salo et al. 2008) compared to $100 \%$ in our study. This, again, suggests that the genual FA finding is particularly characteristic of MA and not tobacco abuse (in 24, lifetime nicotine exposure was permitted for MA subjects, but no details on tobacco use are provided).

Prefrontal white matter and the genu of the corpus callosum are both late-myelinating and, therefore, more vulnerable to damage than the early-myelinating splenium and internal capsule (Yakovlev and Lecours 1967). While it is known that catecholamines (primarily dopamine and serotonin) and acetylcholine can impact oligodendrocyte differentiation and myelination, the exact mechanisms and their impact on early versus late myelination are unclear (Bartzokis 2007; Belachew 1999; Bongarzone 1998; Roy 2007). The genu FA group difference also suggests that the FA differences in other late-myelinating regions are unlikely to be due to more fiber crossing in the MA group (since in the genu, the fibers are parallel), and the underlying process may reflect MA toxicity or preexisting poor myelination.
Hippocampal formation abnormalities

We measured lower FA in MA than in HC subjects, in right perforant path, a white-matter tract not analyzed by earlier studies of MA abuse. Considering our prior observation of morphometric abnormalities in the hippocampus of MA subjects (Thompson et al. 2004), the present finding suggests that white-matter microstructural effects may contribute to neuropathology in the hippocampal formation of MA-dependent subjects. Note that the perforant path is a late-myelinating region (Benes et al. 1994; Arnold and Trojanowski 1996; Benes 1989). In general (with the possible exception of the corona radiata findings), our findings seem to be limited to more vulnerable later-myelinating structures. These results suggest a developmental problem that is either inherent or due to MA. The findings of FA being lower in the MA than the $\mathrm{HC}$ subjects are consistent with gliosis or other white-matter pathology, such as myelin and/or axonal damage or loss.

\section{Sex differences}

With the exception of right perforant path, at every whitematter site where below-normal FA was observed for the overall (male + female) MA group, the same FA effect was manifest for the comparison involving only female subjects. For the comparison involving only male subjects, the MAassociated effect was seen only (and as a trend) in the right perforant path. Thus, overall findings of FA being below $\mathrm{HC}$ values were driven by female MA subjects at all but one site. Observations of low FA reported before (Chung et al. 2007), in contrast, were driven by male MA subjects. Salo et al. (2008) reported no effect of sex on MAassociated below-normal FA in genu corpus callosum, and Kim et al. (2009) reproduced this finding in an all-male sample. Since similar effects on FA were seen in the studies with an excess of male (Chung et al. 2007; Kim et al. 2009) and an excess of female (Salo et al. 2008; present study) MA subjects, these effects apparently occur in both sexes. It also argues against the suggestion that below-normal FA is not observed in white matter of female MA subjects due to neuroprotective effects of estrogen (Chung et al. 2007). Moreover, the failure to find significantly below-normal FA in males in our study and in females previously (Chung et al. 2007) may reflect insufficient numbers of male and female subjects in the two studies, respectively. There were no significant differences between the male and female subsamples of the MA group in years of MA abuse (male $7.4 \pm 6.4$ years, female $8.5 \pm 7.7$ years; $t(21)=0.076$, $p=0.72$ ), age at first abuse (male $25.9 \pm 7.7$ years, female $23.0 \pm 8.9$ years; $t(21)=0.17, p=0.42)$, or years of education (male $12.7 \pm 2.1$ years, female $12.5 \pm 1.6$ years; $t(21)=0.05$, 
$p=0.77)$. Thus, these factors are unlikely to explain the observed sex differences in regional FA.

Correlations of FA with depressive and generalized psychiatric symptoms

Mean scores on the BDI (774\% higher) and BSI Positive Symptom Total $(615 \%$ higher) clinical rating scales were much higher for the MA than for the HC group. This is consistent with earlier findings on MA subjects in the first three weeks of abstinence (McGregor et al. 2005; London et al. 2004), the high lifetime prevalence of depression in individuals who have abused MA (Conway et al. 2006), and the high prevalence of generalized psychiatric symptoms in subjects who engage in MA abuse (Leventhal et al. 2008; Kalechstein et al. 2000; Drake et al. 2008). The present study uncovered correlations between scores on rating scales and FA in left midcaudal superior corona radiata, white matter forming direct two-way connections between frontal cortex and the brain stem and spinal cord. Thus, DTI may join positron emission tomography (London et al. 2004; Sekine et al. 2001) and magnetic resonance spectroscopy (Sekine et al. 2001) as human in vivo neuroimaging modalities with potential to map psychiatric function in MA abuse. As to the functional significance of the specific effects observed, the BSI subscales contributing to the correlation (depression, somatization, obsessive-compulsive, interpersonal sensitivity, paranoid ideation, and phobic anxiety) are all conditions that may entail rumination and cyclic internally verbalized anxiety as a feature. Since the time of Moniz (1936), it has been known that prefrontal leukotomy may alleviate conditions such as anxious neuroses, depression, obsessive-compulsive disorder, and psychosis. In a similar manner, MA-abusing subjects with (especially left hemisphere) prefrontal white-matter FA in the lower range may have sustained damage to neural circuits that support ruminative mental activity resulting in lower scores on the BSI and its subscales. Alternatively, MA-abusing subjects with higher premorbid FA may possess a higher capacity for expressing these symptoms.

Our findings further suggest that regional FA of brain white matter reflects not only frontal executive functions (Wisconsin Card Sorting; 22) and selective attention and response inhibition (Stroop Interference; 23-24) but also mood and psychiatric symptoms. In the other studies cited, regional FA was lower for MA subjects with higher functional impairment (lower task performance). In our study, in contrast, regional FA was higher for MA subjects with more severe mood and psychiatric dysfunction (higher BDI and BSI Positive Symptom Total scores). Thus, even though mean FA was lower for MA than for HC subjects as a group, within the MA group, those subjects reporting worse symptoms registered higher FA values. We do not know the reason for these counter-intuitive results, which may be chance findings; however, we offer that those MA subjects with more intact white matter (higher FA) may be more aware of their own illness and/or be more willing to endorse symptoms. Along these lines, we observed a similar positive correlation of BDI score with gray matter volume in prefrontal cortex in a larger but overlapping sample of MA subjects (A. Morales et al., manuscript in review).

\section{Limitations and strengths}

Limitations of this study include modest sample size and the DTI acquisition protocol (1.5 T, six DTI directions, relatively large voxels). Nonetheless, using this sample size and these relatively gross acquisition parameters, we reproduced and amplified prior regional FA findings. Strengths of this study include defining a relatively narrow period of abstinence for study and the inclusion of samples in which $\mathrm{HC}$ subjects were all tobacco smokers, addressing the specificity of MA effects beyond those of cigarette smoking. This suggests that diminished FA represents a robust feature of white-matter pathology in the brains of humans who abuse MA. Prospective studies are needed to address cause-and-effect issues.

Acknowledgments This work was supported by grants from the National Institute on Drug Abuse (P20DA022539, R01DA020726, R03DA20512, and R21DA023192; EDL and JON) and by the National Center for Research Resources grant MOIRR00865 (UCLA General Clinical Research Center). Additional funding was provided by endowments from the Katherine K. and Thomas P. Pike Chair in Addiction Studies and the Marjorie Green Family Trust (EDL).

Disclosure/conflict of interest The authors report no conflicts of interest.

Open Access This article is distributed under the terms of the Creative Commons Attribution Noncommercial License which permits any noncommercial use, distribution, and reproduction in any medium, provided the original author(s) and source are credited.

\section{References}

Agosta F, Lagania M, Valsasina P, Sala S, Dall'Occhio L, Sormani MP, Judica E, Filippi M (2007) Evidence for cervical cord tissue disorganisation with aging by diffusion tensor MRI. NeuroImage 36:728-735

Ahrens ET, Laidlaw DH, Readhead C, Brosnan CF, Fraser SE, Jacobs RE (1998) MR microscopy of transgenic mice that spontaneously acquire experimental allergic encephalomyelitis. Magn Reson Med 40:119-132

Arnold SE, Trojanowski JQ (1996) Human fetal hippocampal development: I. Cytoarchitecture, myeloarchitecture, and neuronal morphologic features. J Comp Neurol 367(2):274-292

Aron AR, Behrens TE, Smith S, Frank MJ, Poldrack RA (2007) Triangulating a cognitive control network using diffusion- 
weighted magnetic resonance imaging (MRI) and functional MRI. J Neurosci 27(14):3743-3752

Bae SC, Lyoo IK, Sung YH, Yoo J, Chung A, Yoon SJ, Kim DJ, Hwang J, Kim SJ, Renshaw PF (2006) Increased white matter hyperintensities in male methamphetamine abusers. Drug Alcohol Depend 81:83-88

Baicy K, London ED (2007) Corticolimbic dysregulation and chronic methamphetamine abuse. Addiction 102(Suppl 1):5-15

Barr AM, Panenka WJ, MacEwan GW, Thornton AE, Lang DJ, Honer WG, Lecomte T (2006) The need for speed: an update on methamphetamine addiction. J Psychiatry Neurosci 31(5):301-313

Bartzokis G (2007) Acetylcholinesterase inhibitors may improve myelin integrity. Biol Psychiatry 62:294-301

Bartzokis G, Beckson M, Lu PH, Edwards N, Rapoport R, Wiseman E, Bridge P (2000) Age-related brain volume reductions in amphetamine and cocaine addicts and normal controls: implications for addiction research. Psychiatry Research: Neuroimaging 98:93-102

Basser PJ (1995) Inferring microstructural features and the physiological state of tissues from diffusion-weighted images. NMR Biomed 8(7-8):333-344

Bastin ME, Clayden JD, Pattie A, Gerrish IF, Wardlaw JM, Deary IJ (2009) Diffusion tensor and magnetization transfer MRI measurements of periventricular white matter hyperintensities in old age. Neurobiol Aging 30:125-136

Beck AT, Ward CH, Mendelson M, Mock J, Erbaugh J (1961) An inventory for measuring depression. Arch Gen Psychiatry 4:561-571

Behrens TEJ, Woolrich MW, Jenkinson M, Johansen-Berg H, Nunes RG, Clare S, Matthews PM, Brady JM, Smith SM (2003) Characterization and propagation of uncertainty in diffusionweighted MR imaging. Magn Reson Med 50:1077-1088

Belachew S (1999) Neurotransmitter-mediated regulation of CNS myelination: a review. Acta Neurol Belg 99:21-31

Benes FM (1989) Myelination of cortical-hippocampal relays during late adolescence. Schizophr Bull 15(4):585-593

Benes FM, Turtle M, Khan Y, Farol P (1994) Myelination of a key relay zone in the hippocampal formation occurs in the human brain during childhood, adolescence, and adulthood. Arch Gen Psychiatry 51(6):477-484

Berman SM, Voytek B, Mandelkern MA, Hassid D, Isaacson A, Monterosso J, Miotto K, Ling W, London ED (2007) Changes in regional cerebral metabolism during early abstinence from chronic methamphetamine abuse. Molec Psychiatry 13(9):897-908

Berman S, O’Neill J, Fears S, Bartzokis G, London ED (2008) Abuse of amphetamines and structural abnormalities in brain. Ann NYAS 1141:195-220

Bongarzone ER (1998) Identification of the dopamine D3 receptor in oligodendrocyte precursors: potential role in regulating differentiation and myelin formation. J Neurosci 18:5344-5353

Brand A, Richter-Landsberg C, Leibfritz D (1993) Multinuclear NMR studies on the energy metabolism of glial and neuronal cells. Dev Neurosci 15:289-298

Brecht ML, von Mayrhauser C, Anglin MD (2000) Predictors of relapse after treatment for methamphetamine use. J Psychoactive Drugs 32:211-220

Chang L, Ernst T, Speck O, Grob CS (2005) Additive effects of HIV and chronic methamphetamine use on brain metabolite abnormalities. Am J Psychiatry 114:65-79

Chang L, Alicata D, Ernst T, Volkow N (2007) Structural and metabolic brain changes in the striatum associated with methamphetamine abuse. Addiction 102(Suppl 1):16-32

Chung A, Lyoo IK, Kim SJ, Hwang J, Bae SC, Sung YH, Sim ME, Song IC, Kim J, Chang KH, Renshaw PF (2007) Decreased frontal white-matter integrity in abstinent methamphetamine abusers. Int J Neuropsychopharmacol 10(6):765-775

Conway KP, Compton W, Stinson FS, Grant BF (2006) Lifetime comorbidity of DSM-IV mood and anxiety disorders and specific drug use disorders: results from the National Epidemiologic Survey on Alcohol and Related Conditions. J Clin Psychiatry 67:247-256

Derogatis LR, Melisaratos N (1983) The brief symptom inventory: an introductory report. Psychol Med 13(3):595-605

Ding B, Chen K-M, Ling H-W, Zheng H, Chai W-M, Li X, Wang T (2008) Diffusion tensor imaging correlates with proton magnetic resonance spectroscopy in posterior cingulate region of patients with Alzheimer's disease. Dement Geriatr Cogn Disord 25:218225

Drake A, Kaye S, McKetin R, Duflou J (2008) Major physical and psychological harms of methamphetamine use. Drug Alcoh Rev 27:253-262

Dumas de la Roque A, Oppenheim C, Chassoux F, Rodrigo S, Beuvon F, Daumas-Duport C, Devaux B, Meder J-F (2005) Diffusion tensor imaging of partial intractable epilepsy. Eur Radiol 15:279_ 285

Ernst T, Chang L, Leonido-Yee M, Speck O (2000) Evidence for longterm neurotoxicity associated with methamphetamine abuse: a 1H MRS study. Neurology 54:1344-1349

Escubedo E, Guitart L, Sureda FX, Jimenez A, Pubill D, Pallas M, Camins A, Camarasa J (1998) Microgliosis and down-regulation of adenosine transporter induced by methamphetamine in rats. Brain Res 814:120-126

First MB, Spitzer RL, Gibbon M, Williams JBW (1995) Structured Clinical Interview for DSM-IV Axis I Disorders, Patient Edition (SCIDP), version 2. New York State Psychiatric Institute, Biometrics Research, New York

Huether G, Zhou D, Rüther E (1997) Causes and consequences of the loss of serotonergic presynapses elicited by the consumption of 3 , 4-methylenedioxymethamphetamine (MDMA, "ecstasy") and its congeners. J Neural Transm 104:771-794

Jenkinson M, Smith S (2001) A global optimisation method for robust affine registration of brain images. Med Image Anal 5:143-156

Jones DK, Lythgoe D, Horsfield MA, Simmons A, Williams SCR, MArkus HS (1999) Characterization of white matter damage in ischemic leukoaraiosis with diffusion tensor MRI. Stroke 30:393-397

Kalechstein AD, Newton TF, Longshore D, Anglin MD, van Gorp WG, Gawin FH (2000) Psychiatric comorbidity of methamphetamine dependence in a forensic sample. J Neuropsychiatry Clin Neurosci 12:480-484

Kim SJ, Lyoo IK, Hwang J, Sung YH, Lee HY, Lee DS, Jeong DU, Renshaw PF (2005) Frontal glucose hypometabolism in abstinent methamphetamine users. Neuropsychopharmacol 30:1383-1391

Kim I-S, Kim Y-T, Song H-J, Lee J-J, Kwon DH, Lee HJ, Kim M-N, Yoo D-S, Chang Y (2009) Reduced corpus callosum white matter microstructural integrity revealed by diffusion tensor eigenvalues in abstinent methamphetamine addicts. NeuroToxicology 30:209-213

Leventhal AM, Kahler CW, Ray LA, Stone K, Young D, Chelminski I, Zimmerman M (2008) Anhedonia and amotivation in psychiatric outpatients with fully remitted stimulant use disorder. Am J Addictions 17:218-233

London ED, Simon SL, Berman SM, Mandelkern MA, Lichtman AM, Bramen J, Shinn AK, Miotto K, Learn J, Dong Y, Matochik JA, Kurian V, Newton T, Woods RP, Rawson R, Ling W (2004) Mood disturbances and regional cerebral metabolic abnormalities in recent methamphetamine abusers. Arch Gen Psychiatry 61:7384

McCann UD, Wong DF, Yokoi F, Villemagne V, Dannals RF, Ricaurte GA (1998) Reduced striatal dopamine transporter density in abstinent methamphetamine and methcathinone users: evidence from positron emission tomography studies with [ 11C]WIN-35, 428. J Neurosci 18:8417-8422 
McGregor C, Srisurapanont M, Jittiwutikarn J, Laobhripatr S, Wongtan T, White JM (2005) The nature, time course and severity of methamphetamine withdrawal. Addiction 100:1320 1329

Moniz E (1936) Essai d'un traitement chirurgical de certaines psychoses. Bull Acad Med (Paris) 115:385-392

Oh JS, Lyoo IK, Sung YH, Hwang J, Kim J, Chung A, Park KS, Kim SJ, Renshaw PF, Song IC (2005) Shape changes of the corpus callosum in abstinent methamphetamine users. Neurosci Lett 384:76-81

Oldfield RC (1971) The assessment and analysis of handedness: the Edinburgh inventory. Neuropsychologia 9(1):97-113

Pandya DN, Seltzer B (1986) The topography of commissural fibers. In: Lepore F, Ptito M, Jasper HH (eds) Two hemispheres-one brain: functions of the corpus callosum. Liss, New York, pp 4774

Payer DE, London ED (2009) Methamphetamine and the brain: findings from brain imaging studies. In: Roll J, Rawson R, Ling W, Shoptaw S (eds). Methamphetamine: A guide for practitioners. Guilford Press, NY, pp. 61-91

Pennypacker KR, Kassed CA, Eidizadeh S, O'Callaghan JP (2000) Brain injury: prolonged induction of transcription factors. Acta Neurobiol Exp (Wars) 60:515-530

Rawson RA, Condon TP (2007) Why do we need an addiction supplement focused on methamphetamine? Addiction 102(Suppl 1): $1-4$

Ricaurte GA, McCann UD (1992) Neurotoxic amphetamine analogues: effects in monkeys and implications for humans. Ann NYAS 648:371-382

Ringman J, O'Neill J, Geschwind D, Medina L, Schaffer B, Varpetian A, Apostolova L, Tseng P, Fitten J, Ortiz F, Cummings J, Bartzokis G (2007) Diffusion tensor imaging in preclinical and presymptomatic carriers of familial Alzheimer's disease mutations. Brain 130:1767-1776

Roh JK, Nam H, Lee MC (1998) A case of central pontine and extrapontine myelinolysis with early hypermetabolism on 18FDGPET scan. J Korean Med Sci 13:99-102

Roy K (2007) Loss of erbB signaling in oligodendrocytes alters myelin and dopaminergic function, a potential mechanism for neuropsychiatric disorders. Proc Natl Acad Sci USA 104:8131-8136

Salo R, Nordahl TE, Buonocore MH, Natsuaki Y, Waters C, Moore CD, Galloway GP, Leamon MH (2008) Cognitive control and white matter callosal microstructure in methamphetaminedependent subjects: a diffusion tensor imaging study. Biol Psychiatry 65(2):122-128

Schmierer K, Wheeler-Kingshott CAM, Tozer DJ, Boulby PA, PArkes HG, Yousry TA, Scaravilli F, Berker GJ, Tofts PS, Miller DH (2008) Quantitative magnetic resonance of post mortem multiple sclerosis brain before and after fixation. Magn Reson Med 59 (2):268-277
Sekine Y, Iyo M, Ouchi Y, Matsunaga T, Tsukada H, Okada H, Yoshikawa E, Futatsubashi M, Takei N, Mori N (2001) Methamphetamine-related psychiatric symptoms and reduced brain dopamine transporters studied with PET. Am J Psychiatry 158:1206-1214

Sekine Y, Minabe Y, Kawai M, Suzuki K, Iyo M, Isoda H, Sakahara H, Ashby CR Jr, Takei N, Mori N (2002) Metabolite alterations in basal ganglia associated with methamphetamine-related psychiatric symptoms: a proton MRS study. Neuropsychopharmacology 27:453-461

Sekine Y, Minabe Y, Ouchi Y, Takei N, Iyo M, Nakamura K, Suzuki K, Tsukada H, Yoshikawa E, Futatsubashi M, Mori N (2003) Association of dopamine transporter loss in the orbitofrontal and dorsolateral prefrontal cortex with methamphetamine-related psychiatric symptoms. Am J Psychiatry 160:1699-1701

Sheng P, Cerruti C, Cadet JL (1994) Methamphetamine (METH) causes reactive gliosis in vitro: attenuation by the ADPribosylation (ADPR) inhibitor, benzamide. Life Sci 55:PL51PL54

Smith SM, Jenkinson M, Woolrich MW, Beckmann CF, Behrens TE, Johansen-Berg $\mathrm{H}$ et al (2004) Advances in functional and structural MR image analysis and implementation as FSL. Neuroimage 23(Suppl 1):S208-S219

Sung YH, Cho SC, Hwang J, Kim SJ, Kim H, Bae S, Kim N, Chang KH, Daniels M, Renshaw PF, Lyoo IK (2007) Relationship between $N$-acetyl-aspartate in gray and white matter of abstinent methamphetamine abusers and their history of drug abuse: a proton magnetic resonance spectroscopy study. Drug Alcohol Depen 88:28-35

Thompson PM, Hayashi K, Simon SL, Geaga JA, Hong MS, Sui Y, Lee JY, Toga AW, Ling W, London ED (2004) Structural abnormalities in the brains of human subjects who use methamphetamine. J Neurosci 24:6028-6036

United Nations Office on Drug and Crime (2003) Ecstasy and amphetamines - global survey 2003. United Nations, Vienna

Volkow ND, Chang L, Wang GJ, Fowler JS, Franceschi D, Sedler MJ, Gatley SJ, Hitzemann R, Ding YS, Wong C, Logan J (2001) Higher cortical and lower subcortical metabolism in detoxified methamphetamine abusers. Am J Psychiatry 158:383-389

Wilson JM, Kalasinsky KS, Levey AI, Bergeron C, Reiber G, Anthony RM, Schmunk GA, Shannak K, Haycock JW, Kish SJ (1996) Striatal dopamine nerve terminal markers in human, chronic methamphetamine users. Nat Med 2:699-703

Yakovlev PI, Lecours A (1967) The myelogenetic cycles of regional maturation of the brain. In: Minkowski A (ed) Regional development of the brain in early life. Blackwell, Oxford, pp 3-70

Zachary RA, Paulson MJ, Gorsuch RL (1985) Estimating WAIS IQ from the Shipley Institute of Living Scale using continuously adjusted age norms. J Clin Psychol 41(6):820-831 